\title{
MAGNETIC PHASE DIAGRAM OF ZINC-BLENDE $\mathrm{Cd}_{1-x} \mathrm{Mn}_{x} \mathrm{Se}^{*}$
}

\author{
M. SAWICKI \\ Institute of Physics, Polish Academy of Sciences \\ Al. Lotników 32/46, 02-668 Warszawa, Poland \\ J.K. FuRdyna AND H. Luo ${ }^{\dagger}$ \\ University of Notre Dame, Notre Dame, Indiana, 46556, USA
}

The paper reports first magnetic measurements performed on MBE grown epilayers of zinc-blende $\mathrm{Cd}_{1-x} \mathrm{Mn}_{x}$ Se. For all samples studied in the concentration range $0.24<x<0.63$ we find at low temperatures a history-dependent magnetic response, suggesting a transition to a spin-glass-like state. The central result of the paper is the presentation of a new, complete magnetic phase diagram for this material.

PACS numbers: 75.50.-y, 75.50.Rr, 75.50.Lk

Magnetic properties of diluted magnetic semiconductors (DMSs) have long been of great interest to the scientific community, for they offer practical examples of strongly frustrated, randomly diluted Heisenberg antiferromagnets with predominantly short-range $\mathrm{Mn}-\mathrm{Mn}$ exchange interaction. As a result, depending on $\mathrm{Mn}$ concentration and temperature, these materials can exhibit either paramagnetic, antiferromagnetic, or spin-glass-like behaviour [1]. Earlier studies of magnetic properties of $\mathrm{Cd}_{1-x} \mathrm{Mn}_{x}$ Se were limited to $x \leq 0.5$, since bulk growth restricts $\mathrm{Cd}_{1-x} \mathrm{Mn}_{x}$ Se to the hexagonal wurtzite phase with $0 \leq x \leq 0.5$. For all available Mn concentrations, low field $(H \leq 50$ Oe) SQUID measurements showed, below the so-called freezing temperature $T_{f}$, a behaviour of tire magnetization $M$ typical of a spin glass [2-4]. Namely, a pronounced difference occurs between field-cooled (FC) and zero-field-cooled (ZFC) magnetization. This is associated with a maximum observed on ZFC magnetization, whose temperature position coincides with that temperature where both $\mathrm{ZFC}$ and FC magnetizations begin to differ. Moreover, when after a FC measurement the external field is switched off, a thermoremanent magnetization (TRM) is observed, which according to the principle of superposition equals the difference $M_{\mathrm{FC}}-M_{\mathrm{ZFC}}$, and therefore vanishes as $T \rightarrow T_{\mathbf{f}}$.

*Work supported by the grant 2 P03 B 06708 from the Committee for Scientific Research (Poland) and at Notre Dame, by the U.S. National Science Foundation Grant DMR 9221390.

$\dagger$ Present address: State University of New York at Buffalo, Buffalo, New York, 14260, USA. 
The limitations on alloy composition in $\mathrm{Cd}_{1-x} \mathrm{Mn}_{x}$ Se have been recently overcome by using the molecular beam epitaxy (MBE). This technique permits the controlled growth of a single phase, zinc-blende material, thus creating a "new" DMS alloy: cubic CdMnSe. And, what is even more exciting, these alloys can be grown at Mn concentration where bulk growth produces mixed structural phases. About $1 \mu \mathrm{m}$ thick epilayers of $\mathrm{Cd}_{1-x} \mathrm{Mn}_{x}$ Se were grown directly on $\mathrm{GaAs}(100)$ [5]. Four layers, with $x=0.40,0.54,0.70$ and 0.75 were investigated by neutron diffraction [6], showing in $x \geq 0.70$ samples the presence of antiferromagnetic long-range order. However, epilayers with $x=0.40$ and 0.50 did not show evidence of magnetic neutron diffraction, which was accounted for by too small thickness of the layers. Therefore, to gain more information on magnetic ordering in cubic $\mathrm{Cd}_{1-x} \mathrm{Mn}_{x} \mathrm{Se}$, we undertook an investigation of magnetization on five samples, with Mn concentrations ranging from $x=0.24$, through $0.42,0.47$, and 0.49 to $x=0.63$. The $\mathrm{Mn}$ concentration was established by X-ray analysis, using a $10 \mathrm{keV}$ electron beam which, despite the small thickness of all layers, could provide us with very accurate measures of $x$. Here the absence of any buffer layer containing either $\mathrm{Cd}$ or Se proved to be very convenient. Such thin layers do not provide much magnetic response, so in order to gain in the magnetic signal, about $1 \mathrm{~cm}^{2}$ of each epilayer was used to prepare the specimen. Each layer was cleaved into approximately $3 \times 4 \mathrm{~mm}^{2}$ shapes, and most of the diamagnetic GaAs substrate was removed by means of mechanical grinding. The pieces prepared in this way were glued together using thinned GE varnish, and such sandwiches were placed on the sample holder, with the layer planes oriented parallel to the magnetic field direction.
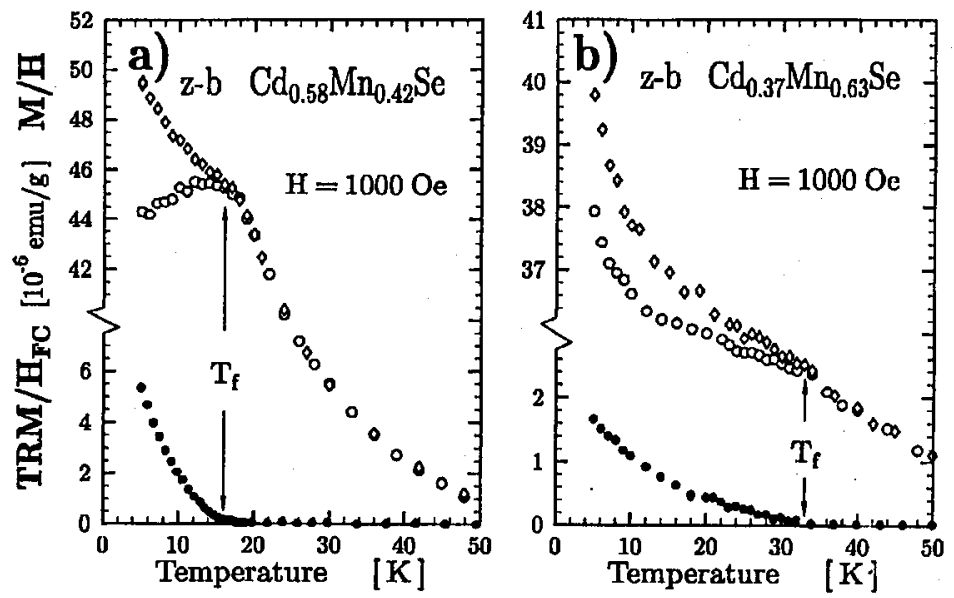

Fig. 1. Temperature dependence of magnetic susceptibility $(M / H)$ and thermoremanent magnetization (TRM/ $H_{\mathrm{FC}}$ ) for zinc-blende $\mathrm{Cd}_{1-x} \mathrm{Mn}_{x}$ Se epilayers: (a) $x=0.42$, and (b) $x=0.63$. Open circles: zero-field-cooled; open diamonds: field-cooled measurements. Bullets: TRM. $H_{\mathrm{FC}}=H_{\mathrm{ZFC}}=H=1000$ Oe. Note that (i) the $Y$ axis is split for better presentation of low temperature behaviour of both $\chi$ and TRM; (ii) TRM for $x=0.63$ is three times weaker than that for $x=0.42$. 
The measurements of temperature dependent magnetization were performed in a home-made rf-biased SQUID magnetometer system equipped with a variable temperature insert. An external DC magnetic field of $H=1 \mathrm{kOe}$ was applied during ZFC and FC measurements. The magnetic susceptibility $\chi(T)$ was obtained from the relation $M(T)=\chi(T) H$, where $M(T)$ denotes magnetic moment $m(T)$ per unit volume of the $\mathrm{Cd}_{1-x} \mathrm{Mn}_{x}$ Se layer only, i.e., after correcting the signal for the diamagnetic contribution from the substrate. Each ZFC-FC cycle of measurements was followed by the measurement of the temperature dependence of TRM. It is worth noting that TRM measurements are generally less demanding to perform, and provide a better estimate of $T_{\mathrm{f}}$, particularly during studies of samples showing such a generally weak magnetic response [7].

Figure 1 shows low temperature results for the ZFC and FC susceptibilities for $x=0.42$ (Fig. 1a) and $x=0.63$ (Fig. 1b). The temperature dependence of the TRM for these two samples is also shown at the bottom of each figure. It must be emphasized that all samples studied showed such irreversible behaviour, typical of the transition to a glassy state: the difference between ZFC and FC data occurs at that temperature where the TRM disappears. Above this temperature, all systems behaved paramagnetically. We also find absolute values of the magnetic susceptibility to be very close to those reported for bulk samples. Specifically, we find that for samples with similar $x$ the $\chi\left(T_{\mathrm{f}}\right)$ values differ by less than about $15 \%$. Certainly, more data are needed to draw a meaningful physical conclusion, but this fact suggests that the correct procedure was used to extract absolute values of magnetic susceptibility for these layers.

These results, combined with the results of the elastic neutron scattering measurements $[6,8]$, allow us to present a new and, so far, most complete magnetic phase diagram for zinc-blende $\mathrm{Cd}_{1-x} \mathrm{Mn}_{x} \mathrm{Se}$, as shown in Fig. 2. We find that for $x>0.45$ the $\mathrm{T}_{\mathrm{f}}$ values for zinc-blende $\mathrm{Cd}_{1-x} \mathrm{Mn}_{x} \mathrm{Se}$ are considerably higher than those found in wurtzite (bulk) $\mathrm{Cd}_{1-x} \mathrm{Mn}_{x}$ Se $[2,3]$. We may expect this tendency to be even stronger, since our results were obtained at higher magnetic field, which is expected to shift $\mathrm{T}_{\mathrm{f}}$ slightly downwards in temperature [9]. Below $x=0.45$, our only one experimental point (for $x=0.24$ ) matches the results of Amarasekara et al. [3]. However, both sets of points have greater values than the points obtained by Oseroff [2]. In the high $x$ range of the diagram, we note that the results from magnetic measurements do not smoothly merge into those from neutron diffraction studies. However, at this moment we are not in the position to unambiguously claim that two different slopes of the $T_{0}(x)$ dependence are indeed observed, a low slope for $x<0.65$, and a much higher one for $x>0.65$, as reported for CdMnTe [10]. On the contrary, since the $\mathrm{Mn}$ concentration in layers studied in [6] has not been confirmed by other method, and that $T_{0}(x=1)$ point comes from the investigation of highly strained, very thin layers [8], we feel that the exact $T_{0}$ dependence in CdMnSe for $x>0.65$ is yet to be clarified. Therefore, a smooth monotonic dependence of $T_{0}$ on $x$ such as that in ZnMnTe [11], may still be observed in CdMnSe.

In conclusion, in this paper we summarized our results of DC magnetic investigations of low temperature properties of MBE-grown zinc-blende $\mathrm{Cd}_{1-x} \mathrm{Mn}_{x} \mathrm{Se}$ epilayers. We detected a characteristic spin-glass-like irreversible behaviour for 


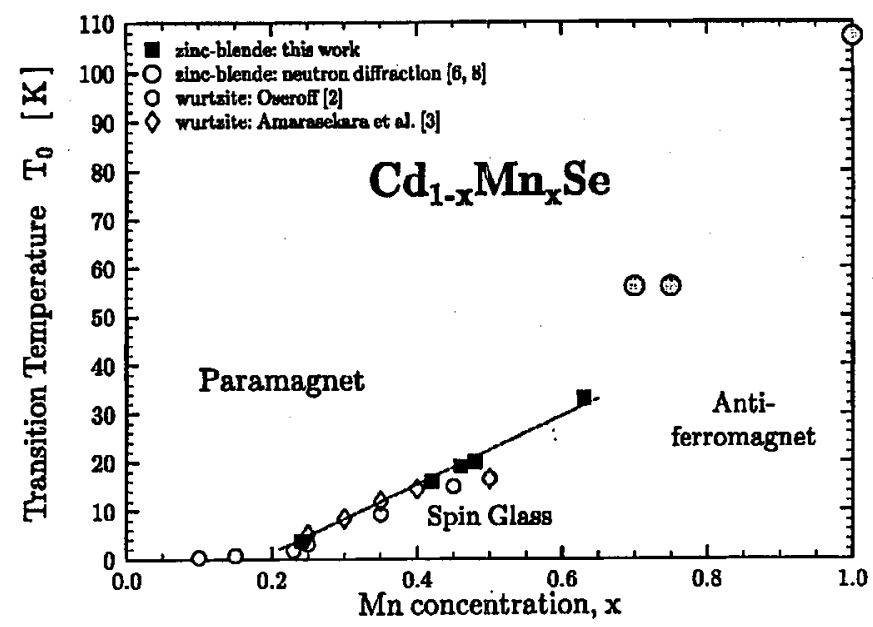

Fig. 2. Magnetic phase diagram for zinc-blende $\mathrm{Cd}_{1-x} \mathrm{Mn}_{x}$ Se obtained from DC magnetic measurements ( $H=1000 \mathrm{Oe}$, full squares - this paper) and neutron-diffraction studies (shaded points - $[6,8]$ ) on MBE-grown epilayers. Transition temperatures observed for bulk samples (open symbols) are shown for comparison. The portion of the boundary between paramagnetic and frozen phases which we consider as firmly established is indicated by the dashed line.

$0.24<x<0.63$. On the basis of this, a new (so far the most complete) magnetic phase diagram for this material $(x<0.65)$ has been proposed.

\section{References}

[1] J.K. Furdyna, J. Appl. Phys. 64, R29 (1988) and references therein.

[2] S.B. Oseroff, Phys. Rev. B 25, 6584 (1982).

[3] C.D. Amarasekara, R.R. Gałąza, Y.Q. Yang, P.H. Keesom, Phys. Rev. B 27, 2868 (1983).

[4] M.A. Novak, O.G. Symko, D.J. Zheng, S. Oseroff, Physica B 126, 469 (1984).

[5] N. Samarth, H. Luo, J.K. Furdyna, Y.R. Lee, R.G. Alonso, E.K. Suh, A.K. Ramdas, S.B. Qadri, N. Otsuka, Surf. Sci. 228, 226 (1990).

[6] T.M. Giebułtowicz, P. Kłosowski, N. Samarth, H. Luo, J.J. Rhyne, J.K. Furdyna, Phys. Rev. B 42, 2582 (1990).

[7] M. Sawicki, P.A.J. de Groot, M.A. Brummell, G.J. Tomka, D.E. Ashenford, B. Lunn, Acta Phys. Pol. A 84, 745 (1993).

[8] P. Kłosowski, T.M. Giebułtowicz, J.J. Rhyne, N. Samarth, H. Luo, J.K. Furdyna, J. Appl. Phys. 69, 6109 (1991).

[9] E.g. H. Kett, W. Gebhard, U. Krey, J.K. Furdyna, J. Magn. Magn. Mater. 25, 215 (1981).

[10] J. Petruczanis, W. Mac, A. Twardowski, G. Karczewski, A. Zakrzewski, E. Janik, T. Wojtowicz, J. Kossut, Mater. Sci. Forum 182-184, 687 (1995).

[11] T.M. Giebultowicz, P. Kłosowski, N. Samarth, H. Luo, J.K. Furdyna, J.J. Rhyne, Phys. Rev. B 48, 12817 (1993). 